\title{
Highlighting the Role of Infections in the Etiology of Fever of Unknown Origin Pointing out Toxoplasmosis; in Port Said Governorate
}

\author{
Ahmed M. Arnous ${ }^{1}$, Nahla E. Elgammal ${ }^{2}$, Nahed E. Mostafa ${ }^{3}$, Soha A. \\ Elhawari $^{2}$, Marwa A. Salama ${ }^{3}$, Eman M. Fawzy ${ }^{3}$ \\ ${ }^{1}$ Port Said Fever Hospital, Port-Said Governorate, Egypt. \\ ${ }^{2}$ Department of Tropical Medicine, Faculty of Medicine, Zagazig University, Sharkia \\ Governorate, Egypt. \\ ${ }^{3}$ Department of Medical Parasitology, Faculty of Medicine, Zagazig University, \\ Sharkia Governorate, Egypt.
}

Corresponding Author Nahed E Mostafa MD

Mobile:

+201100720092

E mail:

nahoodaah58@gmail. com

Key words: FUO, infectious etiology, human toxoplasmosis, Egypt
Background and study aim: Fever of unknown origin (FUO) is a state of febrile illness for more than three weeks, with a body temperature greater than $38.3{ }^{\circ} \mathrm{C}$ on several occasions and uncertain diagnosis. Conditions blamed for FUO were categorized as infectious diseases, noninfectious inflammatory diseases, neoplasms along with miscellaneous causes. This study aims to identify the infectious causes of FUO counting the parasitic causes in Port-Said Fever Hospital/ Egypt.

Patients and Methods: 341 patients admitted to Port-Said Fever Hospital/ Egypt during the period from November 2017 till May 2018 complaining of prolonged fever $\geq 3$ weeks, without a definite diagnosis, were included in this study. Complete history taking and clinical data including pattern, duration and degree of temperature, in addition to other co-morbidities, radiological examination, laboratory investigations, and clinical evaluation were reported for each patient.

Results: The majority of affected cases were adult males, lived in urban areas, and were in contact with animals.

\section{INTRODUCTION}

Fever of unknown origin (FUO), or fever lacking a definite cause, is an exciting clinical challenge that is omnipresent [1]. Temperature going upwards $38.3^{\circ} \mathrm{C}$ on numerous occasions for 3 weeks or more without definitive diagnosis, is defined as fever of unknown origin
Luncheon and beef were the commonest types of consumed food among the studied population. The uppermost detected infection was tuberculosis (17\%) followed by typhoid and Brucella infection (11.7\% for each), toxoplasmosis and hepatitis were detected in $2.9 \%$ and $1.8 \%$ respectively. After investigations, $5.9 \%$ of the studied group had hepatosplenomegaly and $77.4 \%$ of them presented with lymphocytosis. Clinically, $78.9 \%$ of patients had lymphadenopathy and $1.8 \%$ had jaundice. Additionally, $75.4 \%$ of cases presented with an intermittent fever while $24.6 \%$ with continuous fever. Approximately $57.2 \%$ of them had fever for three weeks and the majority of patients had fever $\geq 39^{\circ} \mathrm{c}$.

Conclusion: Tuberculosis, typhoid fever, and brucellosis signified the major identified infectious causes for FUO in Port Said governorate/Egypt. Toxoplasmosis can be represented as one of FUO infectious causes. Patients with toxoplasmosis either presented with lymphadenopathy or not, all of them had hepatosplenomegaly, high CRP, leucocytosis, and eosinophilia.

(FUO) [2]. The difficulty in diagnosing several cases of FUO may be due to the a wide range of the factors that can influence its final diagnosis [3]. Many scholars identified over 200 conditions to be responsible for FUO [4], they categorize them as infectious diseases, non-infectious inflammatory diseases, neoplasms, and miscellaneous causes 
[5]. Various concerns can influence the diagnosis of FUO. Geographic distribution, demographic data, socioeconomic level, the outlook of clinicians as well as the difference in investigation funds between developed and developing countries may possibly of a great significance [3]. Infectious diseases remain the main cause of FUO particularly in developing countries [6]. Aiming to shed light on the contributing aspects that influence the clinical spectrum of FUO, we carried out this research.

Toxoplasmosis; is a universal, food borne parasitic infection with inconstant symptoms and signs [7]. Toxoplasma gondii is an obligate intracellular parasite that can invade and replicate within all nucleated cells of worm blooded animals and humans [8]. It has a complex life cycle with various stages fluctuating between replicating (tachyzoites) and quiescent (bradyzoites) forms [9].Within the intermediate hosts' cell, the parasite initially replicates as tachyzoites, with an intense increase in number and spread to different body tissues. After a potent immune response, it differentiates to dormant bradyzoites within tissue cysts resides in neurons and skeletal muscle cells [10]. Human infection with Toxoplasma gondii occurs via eating raw or undercooked meat or drinking contaminated water [11], contaminated garden vegetables with oocysts shed by cats, in addition to the congenital infection when a mother is infected early during pregnancy[12]. The serological prevalence of toxoplasmosis, mostly reflecting subclinical chronic infections, is extremely variable. Approximately $25 \%$ of the world's population may be chronically infected with $T$. gondii [13]. In healthy grown-ups, Toxoplasma induces a quite mild infection, with a raised temperature, muscle weakness, and enlarged lymph nodes. FUO and weakness in the upper legs are among the presenting symptoms in acute toxoplasmosis, which can occur in immune-competent individuals [14]. Acute infection usually resolves quickly, leaving the individual with chronic, subclinical infection [15].

\section{PATIENTS AND METHODS}

\section{Study design:}

Our cross-sectional study incorporated 341 patients admitted to Port-Said Fever Hospital/ Egypt during the period from November 2017 till
May 2018supervised by the Tropical Medicine Department, Faculty of Medicine, Zagazig University/ Egypt.

\section{Patients:}

The current study comprised of 341 patients complaining of fever, including 231 males and 110 females, with an age range of $16-70$ years. Patients fulfilled the inclusion criteria (prolonged fever $\geq 3$ weeks, without definite origin, with /without lymph node enlargement or splenomegaly) were included in our study, while patients with the diagnosed cause of fever were excluded.

\section{Data collection:}

All patients were subjected to complete history taking (age, sex, residence, animal contact, dealing with soil and type of consumed food). Clinical data included (previous hospital admission, pattern, duration, and degree of temperature, in addition to other co-morbidity e.g hypertension, diabetes, renal/ hepatic disease, abortion, medications).

\section{Clinical evaluation \& radiological examination:}

All patients were examined for vital signs, temperature, pallor or jaundice, chest and abdominal examination, abnormal body swellings, lymph node enlargement, and lower limb edema then subjected to radiological imaging including chest X-ray, abdominal and pelvic ultrasound (US) in Port-Said Fever Hospital/ Egypt.

\section{Laboratory investigations:}

The following laboratory investigations were carried out:

- Complete blood picture (CBC), CRP, Liver function tests, kidney function tests, thyroid function tests, blood sugar, stool and urine analysis.

- Detection of serum hepatitis A (HAV)IgM by radioimmunoassay.

- Hepatitis C (HCV) antibodies and hepatitis B surface antigen ( $\mathrm{HBs} \mathrm{Ag}$ ) were detected using an enzyme immunoassay for the qualitative determination of antibodies in human serum / plasma samples by ELISA kits (Diasorin S.p.A. 13040 Saluggia (VERCELLI, Italy).

- Widal test, brucella agglutination test and tuberculin test as well.

- Specific markers as the anti-nuclear antibody (ANA) and anti- double-strand DNA (anti-ds-

Arnous et al., Afro-Egypt J Infect Endem Dis 2020 ;10(3):301-309

https://aeji.journals.ekb.eg/

http://mis.zu.edu.eg/ajied/home.aspx 
DNA) for systemic lupus erythematosus (SLE) were detected in serum using Electrochemiluminescence practicing Cobas e411 (Roche Diagnostic GmbH, Sandhofer Strasse 116, D-68305 Marnheim, Germany).

- Assessment of Anti-Toxoplasma IgM \& IgG antibodies using the Electrochem- iluminescence immunoassay using Cobas e411 (RocheDiagnostic GmbH, SandhoferStrasse 116, D-68305 Marnheim, Germany) guided by the manufacturer's guidelines.

\section{RESULTS}

Table (1): Demographic data of the studied population.

\begin{tabular}{|l|c|c|}
\hline & $\begin{array}{c}\text { No. } \\
(\mathrm{n}=341)\end{array}$ & $\%$ \\
\hline Age & & \\
Adolescent & 35 & $10.3 \%$ \\
Adult & 306 & $89.7 \%$ \\
\hline Sex & & \\
Male & 231 & $67.7 \%$ \\
Female & 110 & $32.3 \%$ \\
\hline Residence & & \\
Urban & 325 & $95.3 \%$ \\
Rural & 16 & $4.7 \%$ \\
\hline Contact with animals & & \\
Yes & 18 & $5.3 \%$ \\
No & 323 & $94.7 \%$ \\
\hline Dealing with soil & & \\
Yes & 9 & $2.6 \%$ \\
No & 332 & $97.4 \%$ \\
\hline
\end{tabular}

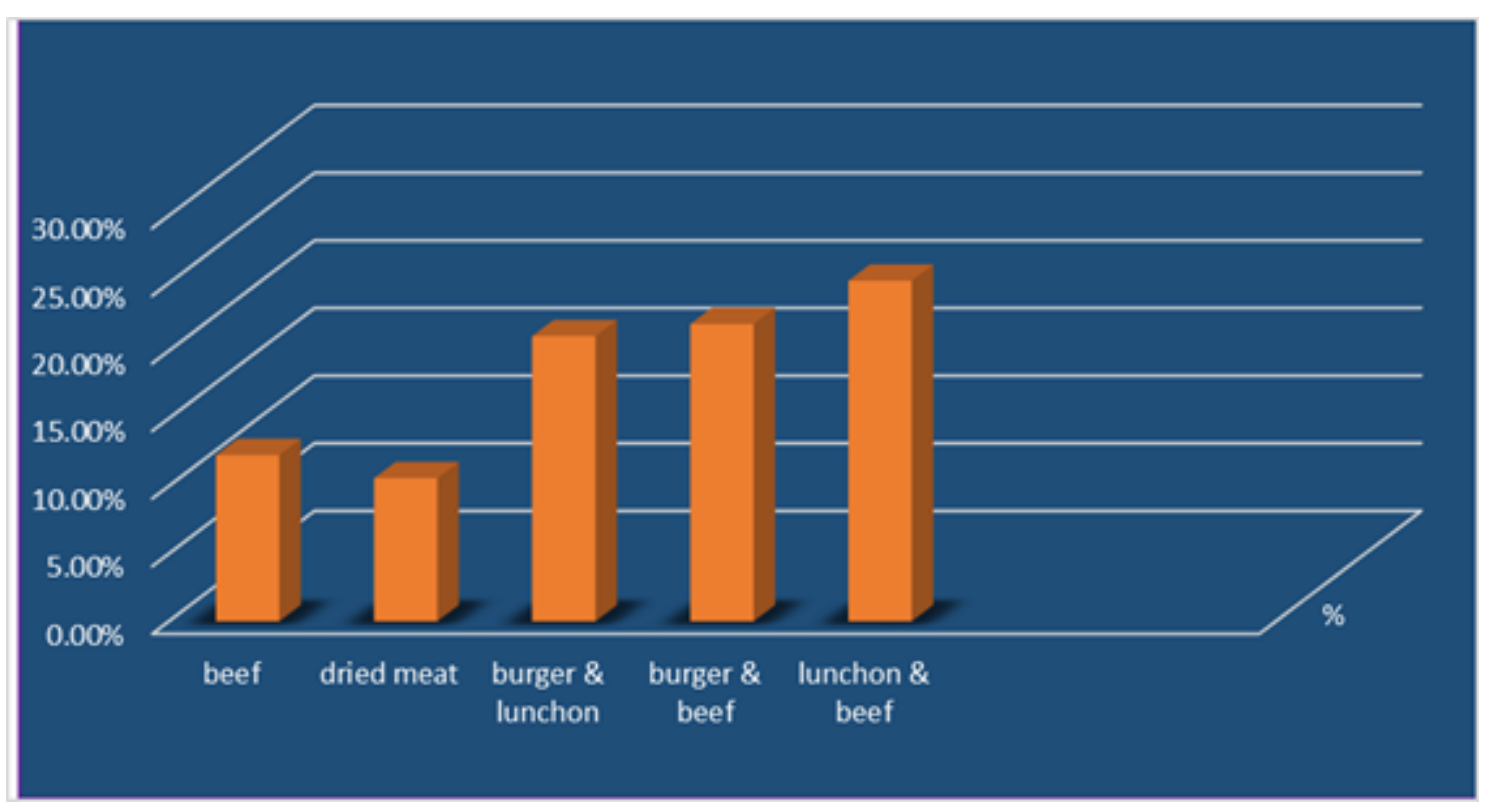

Fig. (1): Bar chart showing different types of consumed food among the studied population. 
Table (2): The ultrasonography, chest X-ray and blood picture among the studied group

\begin{tabular}{|l|c|c|}
\hline & $\begin{array}{c}\text { No. } \\
(\mathrm{n}=341)\end{array}$ & $\%$ \\
\hline US (HSM) & 20 & $5.9 \%$ \\
+ve cases & 321 & $94.1 \%$ \\
-ve cases & & \\
\hline Chest X-ray finding & 25 & $7.3 \%$ \\
+ve X-ray finding & 316 & $92.7 \%$ \\
Free chest & & \\
\hline Blood picture & 16 & $4.7 \%$ \\
normal & 61 & $17.9 \%$ \\
leucocytosis \& eosinophilia & 264 & $77.4 \%$ \\
\hline lymphocytosis & \multicolumn{2}{|}{} \\
\hline
\end{tabular}

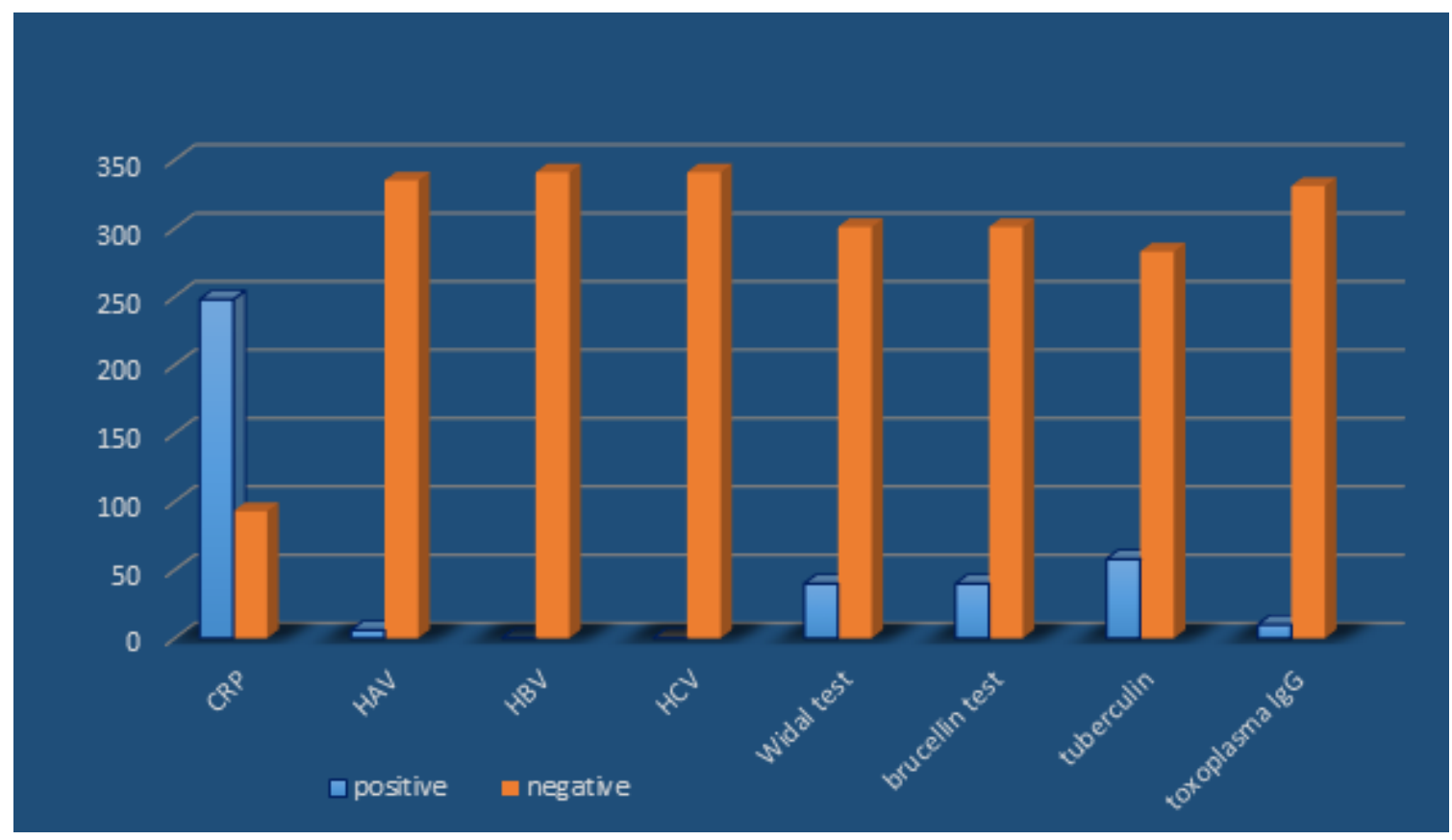

Fig. (2): Bar chart showing lab finding among the studied group.

Table (3): Characteristics of fever among the studied group.

\begin{tabular}{|l|c|c|}
\hline & $\mathrm{N} 0$ & $\%$ \\
$(\mathrm{n}=341)$ & & \\
\hline Pattern of fever & 84 & $24.6 \%$ \\
Continuous & 157 & $46 \%$ \\
Intermittent & 100 & $29.3 \%$ \\
relapsing & 195 & $57.2 \%$ \\
\hline Duration of fever & 146 & $42.8 \%$ \\
$3-4$ weeks & & \\
$\geq 4$ weeks & 10 & $2.9 \%$ \\
\hline Temperature rising & 205 & $60.1 \%$ \\
$38-39^{\circ} \mathrm{c}$ & 126 & $37.0 \%$ \\
$39-40^{\circ} \mathrm{c}$ & \\
$>40^{\circ} \mathrm{c}$ & \multicolumn{2}{|l}{} \\
\hline
\end{tabular}


Table (4): Clinical presentations \& examinations within the studied group.

\begin{tabular}{|l|c|c|}
\hline & $\begin{array}{c}\text { No. } \\
(\mathrm{n}=341)\end{array}$ & $\%$ \\
\hline LN & 269 & $78.9 \%$ \\
Enlarged & 72 & 22.1 \\
Normal size & & \\
\hline Jaundice & 6 & $1.8 \%$ \\
Yes \\
no & 335 & $98.2 \%$ \\
\hline treatment with antibiotics & & \\
Yes & 257 & $75.4 \%$ \\
no & 84 & $24.6 \%$ \\
\hline
\end{tabular}

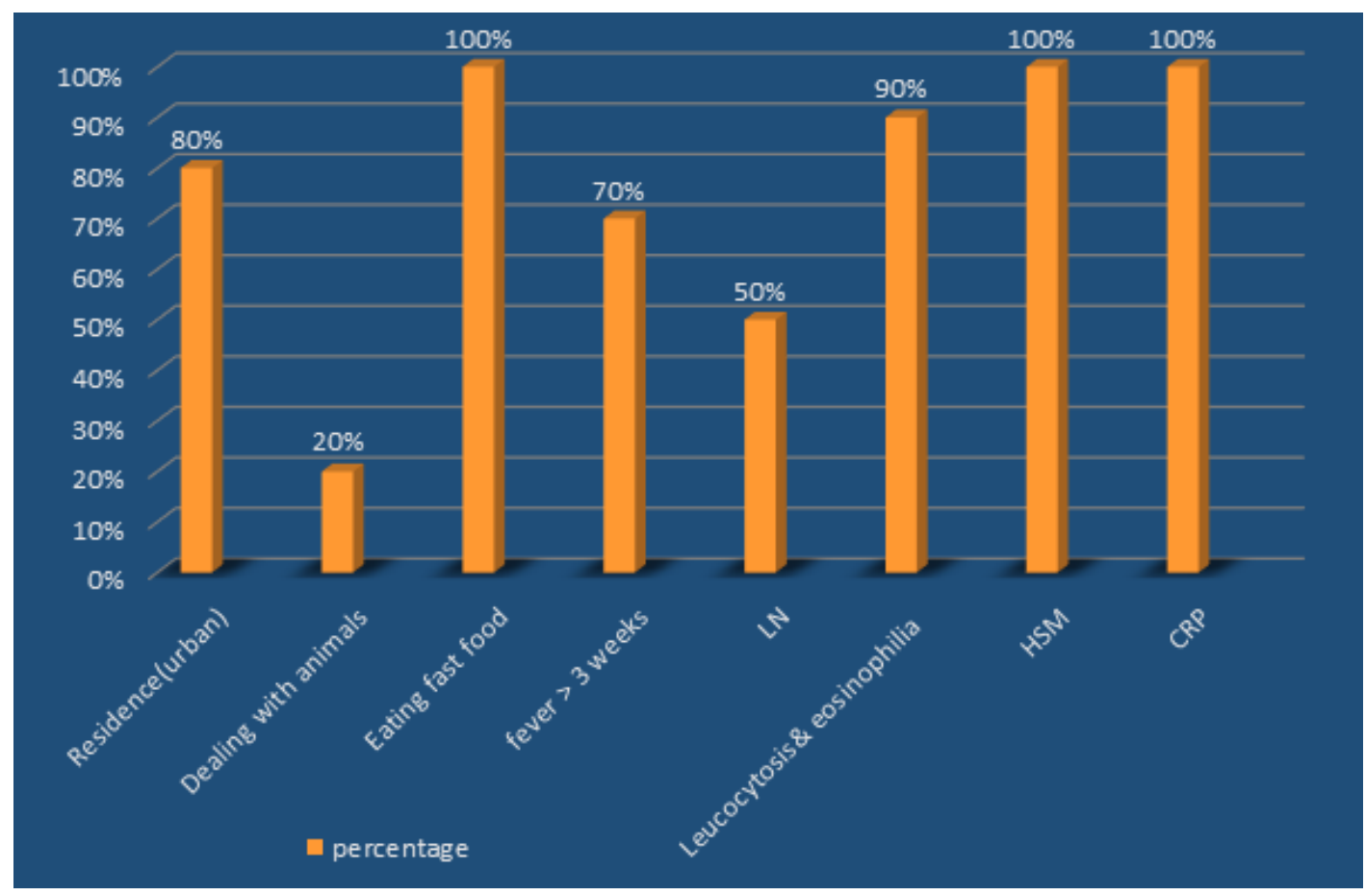

Fig. (4): Bar chart shows demography, types of consumed food, clinical presentation and investigations among only toxoplasmosis cases.

\section{DISCUSSION}

FUO is a state of febrile illness for more than three weeks, with a body temperature greater than $38.3^{\circ} \mathrm{C}$ on several occasions and uncertain diagnosis[16]. The foremost causes are the infectious, the neoplastic, and rheumatic. The diagnosis is based on good clinical history, the physical examination of these patients, and the proper investigations [1].

Studying the demographic data of the studied population in this work presented in the table (1), we observed that the majority of affected cases were adults. Almost two-thirds of the studied participants were males. The majority of them lived in urban areas, and at most 5\% of them had contact with animals. Furthermore, only 9 patients were dealing with soil. Santana et al. [17] emphasized that elderly patients constituted the most represented among FUO cases. Others proved that females are generally more frequently affected than males, as females have increased immune reactivity, resulting in resistance to infectious and some non-infectious diseases. However, it is possible that this pronounced immune reactivity makes women more prone to developing autoimmune diseases [18]. Contact with animals or birds is important risk factors for acquiring infections that may cause FUO [19].

The majority of our cases lived in urban areas, this may be as a result of urbanization and the general increase of quality and duration of life 
that may lead to the detection of some feverish patients who would not receive medical care in a rural area.

Concerning types of consumed food among the studied population (figure 1), luncheon and beef were the commonest type of consumed food followed by a burger and beef $(25.2 \%$ and $22 \%$ respectively) and the least consumed type was dried meat (10.6\%). We suppose that this kind of food may contribute to Salmonella or Toxoplasma infection, which are common with poor sanitation in developing countries.

Owing to high costs, no specific investigational flow protocol for the diagnosis of FUO in Egypt is followed. Only initial investigations are done, together with the history and clinical findings that may guide the diagnosis [20].

Fever of unknown origin remains a difficult diagnostic challenge because over 200 disorders are in the differential diagnosis [21]. The diagnostic procedure is divided into multiple stages. The first includes ESR, C-reactive protein, complete blood count, renal and liver function tests, creatine phosphokinase, lactate dehydrogenase, ferritin, serum electrophoresis, antinuclear antibodies, rheumatoid factors, urine analysis, blood and urine cultures, radiography of chest, pelvi-abdominal ultrasonography were included as well. The second requires more specific radiological, serological, and microbiological tests. More invasive techniques are used, as bone marrow aspiration, liver biopsy, scintigraphy, or emission tomography, in addition to molecular tools [22]. The diagnostic work-up In Taiwan [23] begins with schedule hematological and biochemical and radiological investigations for all patients. In Saudi Arabia [24], A single diagnostic work-up is performed for all FUO cases involving the majority of mentioned investigations. The variability in diagnostic work-up give you an idea about the lack of integrated approach and strategy for handling the FUO cases. Despite considerable development in the imaging, serological, and immune-histopathological investigations, the task of diagnosis is often difficult and cannot be achieved in up to $50 \%$ of the cases [25].

Lab findings among the studied group in the present work (Figure 2) revealed that about $72.7 \%$ of the studied participants had positive CRP. The number of +ve CRP detected cases were more than the number of infectious diseases included in this work disclosed that there were some patients suffering from other infections, collagen or tissue destructing disorders not investigated in our study. The uppermost detected infection in our work was tuberculosis (17\%) followed by Salmonella and Brucella infections (11.7\% for each). Furthermore, toxoplasmosis and hepatitis were detected in $2.9 \%$ and $1.8 \%$ respectively. A study done by Lamiaa et al. [26] revealed that bacterial infection was on the top of FUO causes, as 50\% of patients had bacterial infections: salmonellosis in $15.6 \%$ of patients, brucellosis in $11.1 \%$ of patients, tuberculosis peritonitis in $10 \%$ patients, pyelonephritis in $7.7 \%$ of patients, pyogenic liver abscesses in of $3.3 \%$ patients, and tuberculosis lymphadenitis in $2.2 \%$ of patients. Moreover, Mir et al. [27] enrolled 91 patients with classical pyrexia unknown origin(PUO), infections were the most common cause (44\%) of PUO, followed by malignancies $(12 \%)$, and connective tissue disorders (12\%).

Investigations among the studied group (table 2) disclosed that $5.9 \%$ of the studied group had hepatosplenomegaly detected by the US. Almost $77.4 \%$ of them presented with lymphocytosis. We cannot exclude the possibility that the falsepositive tuberculin test may have accounted for some of the TB cases in persons with normal CXRs, as Pepper et al. [28] accounted that the most important finding in their study was the high rate of normal chest X-ray (CXR) among persons with culture-confirmed Pulmonary TB (PTB). Also, renal failure patients are known to be associated with an increased frequency of atypical radiographic presentations of PTB, including normal CXR [29]. However, DNA fingerprinting must be performed when laboratory cross-contamination was suspected, false-positive tests or cultures were therefore unlikely. Almost $77.4 \%$ of patients in the present study presented with lymphocytosis. Similar to our findings Lamiaa et al. [26] found that 28 (31.12\%) out of 90 patients had leukocytosis, whereas leucopenia was presented in 12 (13.33\%) patients. Also, 22 (24.4\%) patients had hepatosplenomegaly. Isolated hepatomegaly could signify Q fever, typhoid/enteric fever and brucellosis. Isolated splenomegaly could be a clue for miliary TB, Epstein-Barr virus, and cytomegalovirus [21].

Concerning the pattern of fever in our studied group, it was noticed that approximately, $46 \%$ of patients presented with intermittent fever, $24.6 \%$ 
presented with continuous fever, and $29.3 \%$ presented with relapsing fever. Around $57.2 \%$ of them had fever lasted for three weeks and the majority of patients had fever $\geq 39^{\circ} \mathrm{c}$ (table 3 ). Among patients who presented with intermittent fever, $10.1 \%$ of patients were diagnosed as salmonellosis, $10.1 \%$ of them as brucellosis, $9.5 \%$ of them as tuberculosis, $3.1 \%$ as toxoplasmosis, and the rest of patients diagnosis could not be reached.

Amidst the patients who presented with continuous fever, $9.5 \%$ of them were diagnosed as salmonellosis, $9.5 \%$ of them as brucellosis, $39.2 \%$ of them as tuberculosis, $5.9 \%$ of them as toxoplasmosis, and the rest of patients diagnosis could not be reached. In dispersion through patients who presented with relapsing fever, $16 \%$ of them were diagnosed as salmonellosis, $16 \%$ of them as brucellosis, $10 \%$ of them as tuberculosis and, the rest of patient's diagnosis could not be reached. Nonspecific presentations and the empirical antibiotic therapies may be the most common reasons for a delay in diagnosis or the undiagnosed cases. In agreement with our results, it was found that $58.3 \%$ of cases were presented with a continuous pattern of fever, while $16 \%$ presented with a remittent pattern, $23.2 \%$ showed intermittent fever, and $1.6 \%$ had a relapsing fever [29]. Also, Lamiaa et al. [26] found that patients who presented with continuous fever were due to salmonellosis, pyelonephritis, and cytomegalovirus, other patients were undiagnosed.

Clinical presentations and examinations of the studied group in the table (4), disclosed about $78.9 \%$ of patients with lymphadenopathy and $1.8 \%$ had jaundice. Besides, about $75.4 \%$ of patients received antibiotics. Under the same circumstances, it was accounted that lymphadenopathy is a useful key feature in the diagnosis of FUO, especially cervical lymphadenopathy [30]. FUO with lymphadenopathy must be considered as a separate entity, mostly represented by malignancies and granulomatosis due to infections or malignancy-related origin [31], in addition to toxoplasmosis [32]

In our research, the co-morbidities signified, $2.6 \%$ of patients with diabetes mellitus, $1.5 \%$ with hypertension, and $1.2 \%$ had the renal disorder.

Poring over demography, types of consumed food, clinical presentation, and investigations of the only cases with toxoplasmosis (figure 3), fever among toxoplasmosis patients were more in adults living in urban areas and dealing with animals without difference between males \& females. The most commonly consumed food was undercooked meat and Burger \& luncheon. They were presented with a continuous or intermittent fever between $39-40^{\circ} \mathrm{c}$. They either presented with lymphadenopathy or not, but all of them had hepatosplenomegaly and high CRP and leukocytosis \& eosinophilia. In agreement with us, Cunha et al. [21] also stated that contact with animals in patients presented with FUO must raise the suspicion of toxoplasmosis, brucellosis, cat-scratch disease, and leptospirosis.

\section{CONCLUSION}

Tuberculosis, typhoid fever, and brucellosis were the main infectious causes in Port Said governorate/Egypt, afterward toxoplasmosis. Most of patients with toxoplasmosis were presented with lymphadenopathy, all of them had hepatosplenomegaly, high CRP, leucocytosis, and eosinophilia. Lymph node, bone marrow and liver biopsies are recommended in future studies beside, CT MRI and PET scan as more advanced investigations will help in declaring the definitive diagnosis of fever.

\section{Ethical respects:}

All participants were informed about the study, and written consent was obtained. An agreement of our study was obtained from the Institutional Review Board (IRB) Unit, Faculty of Medicine, Zagazig University, Egypt (no. 4056) meeting the requirements of the Helsinki Declaration.

\section{Financial support and sponsorship}

Nil.

\section{Conflicts of interest}

There are no conflicts of interest.

\section{REFERENCES}

1. Santana LF, Rodrigues MD, de Araújo Silva MD, Cordeiro de Brito RV, Nicacio JM, Duarte RM, Gomes OV. Fever of unknown origin - a literature review. Rev Assoc Med Bras 2019; 65(8):1109-1115. 
2. Zhao M, Ruan Q, Xing M, Wei S, Xu D, Wu Z, et al. A Diagnostic Tool for Identification of Etiologies of Fever of Unknown Origin in Adult Patients. Curr Med Sci 2019; 39(4):589-596.

3. Fusco FM, Pisapia R, Nardiello S, Cicala SD, Gaeta GB, Brancaccio G. Fever of unknown origin (FUO): which are the factors influencing the final diagnosis? A 2005-2015 systematic review. BMC Inf Dis 2019; 19(1):653.

4. Mourad O, Palda V, Detsky AS. A comprehensive evidence-based approach to fever of unknown origin. Arch Intern Med 2003; 163:545-551.

5. 5. Gaeta GB, Fusco FM, Nardiello S. Fever of unknown origin: a systematic review of the literature for 1995-2004. Nucl Med Commun 2006; 27(3):205-211.

6. Tu JC, Ping Z, Li XJ, Sun Y, Hui-Yuan S, Wanget $\mathrm{C}$, et al. Clinical Aetiologies of Fever of Unknown Origin in 500 Cases. Zhongguo $\mathrm{Yi}$ ХиеКеХие Yиаn ХиеВаo (Chinese) 2015; 37(3):348-351.

7. Basit KA, Nasir S, Vohra E, Shazlee MK. Toxoplasmosis in an Immunocompetent Patient. Pak J Med Sci 2018; 34(6):1579-1581.

8. Iqbal J, Al-Awadhi M. Toxoplasmosis: Role of Cytokines in Disease Modulation \& Tissue Pathology. Ann ClinPathol 2016;4(7): 1090.

9. Sullivan WJ, Jeffers, V. Mechanisms of Toxoplasma gondii persistence and latency. FEMS Microbiol Rev 2012; 36: 717-733.

10. Dubey JP. Tissue cyst tropism in Toxoplasma gondii: a comparison of tissue cyst formation in organs of cats, and rodents fed oocysts. Parasitology 1997; 115:15-20.

11. Dubey JP. Toxoplasmosis of Animals and Humans, second ed. CRC Press, Boca Raton Florida 2010; 1-313.

12. Hakimi MA, Olias P, Sibley LD. Toxoplasma Effectors Targeting Host Signaling and Transcription. ClinMicrobiol Rev 2017; 30 (3):615-645.

13. Pappas G, Roussos N, Falagas ME. Toxoplasmosis snapshots: global status of Toxoplasma gondii seroprevalence and implications for pregnancy and congenital toxoplasmosis. Int J Parasitol 2009; 39:13851394.

14. Mentink JF, van Rijn M, Dees A. A fever of unknown origin as a presenting symptom in toxoplasmosis: Back to earth. Int J Case Rep Images 2017; 8(1):73-76.
15. Petersen CCH. The Functional Organization of the Barrel Cortex. Neuron 2007; 25; 56(2):33955.

16. Petersdorf RG, Beeson PB. Fever of unexplained origin: report on 100 cases. Medicine (Baltimore) 1961; 40:1-30.

17. Alavi SM, Nadimi M, Sefidgaran G, Papi $\mathrm{MH}$, Zamani GA. Clinical spectrum and diagnostic tools of fever of unknown origin among hospitalized patients in Razi Hospital (2006-2008). Ahvaz. Jundishapur J Microbiol 2011; 2: 152-157.

18. Ngo ST, Steyn FJ, McCombe PA. Gender differences in autoimmune disease. Front Neuro Endocrinol 2014; 35: 347-369.

19. Kabapy AF, Kotkat AM, Shatat HZ, Abd El Wahab EW. Clinico-epidemiological profile of fever of unknown origin in an Egyptian setting: A hospital-based study (2009-2010). J Infect Dev Ctries 2016 Jan 31; 10(1): 30-42.

20. Montasser MF, Abdelkader NA, Montasser IF, El Khouly AM. Changing the face of fever of unknown origin in Egypt: a single hospital study. Braz J Infect Dis 2015;19(3):334-335

21. Cunha BA, Lortholary O, Cunha CB. Fever of unknown origin: a clinical approach. Am J Med 2015; 128(10):1138.e1-1138.e15.

22. Zenone $\mathrm{T}$ (2006) Fever of unknown origin in adults: evaluation of 144 cases in a nonuniversity hospital. Scand J Infect Dis 38: 632638.

23. Chin C, Chen YS, Lee SSJ, Wann SR, Lin $\mathrm{HH}$, Lin WR, et al. Fever of unknown origin in Taiwan. Infection 2006; 34: 75-80.

24. Moawad MA, Bassil H, Elsherif M, Ibrahim A, Elnaggar M, Edathodu $\mathrm{J}$, et al. Fever of unknown origin: 98 cases from Saudi Arabia. Ann Saudi Med 2010; 30: 289.

25. Bleeker-Rovers CP, Vos FJ, de Kleijn EM, Mudde AH, Dofferhoff TS, Richter C, et al. A prospective multicenter study on fever of unknown origin: the yield of a structured diagnostic protocol. Medicine (Baltimore) 2007; 86(1):26-38.

26. Lamiaa SS, Abeer SEA, Zainab GM. Evaluation of different patterns and etiologies of classic form of pyrexia of unknown origin in Assiut University fever unit. $J$ of CurrMedi Res and Prac 2019; 4:98-103.

27. Mir T, Dhobi GN, Koul AN, Saleh T. Clinical profile of classical FUO. Caspian J Intern Med 2014; 5:35-39. 
28. Pepper T, Joseph P, Mwenya C, McKee G. Normal chest radiography in pulmonary tuberculosis: Implications for obtaining respiratory specimen cultures. Int $J$ Tub and Lung Dis 2008; 12(4):397-403.

29. Fang HC, Lee PT, Chen CL, Wu MJ, Chou KJ, Chung H M. Tuberculosis in patients with endstage renal disease. Int J Tuberc Lung Dis 2004; 8: 92-97.

30. Takeda R, Mizooka M, Kobayashi M, Kishikawa N, Yokobayashi K, Kanno K, et al. Key diagnostic features of fever of unknown origin: Medical history and physical findings. $J$ Gen Fam Med 2017; 23;18(3):131-134.
31. Bentz HM, Dupond JL. Diagnostic value of lymphadenopathy associated with fever and inflammation of unknown origin: A study of 69 patients. Rev Méd Interne 2011; 32(8):461-466.

32. Pei Lin, L. Jeffrey Medeiros: Hematopoietic Lesions. Diagnostic Surgical Pathology of the Head and Neck (Second Edition), 2009. 